\title{
REPRESENTATION AND APPROXIMATION BY SERIES OF CONTINUOUS FUNCTIONS
}

\author{
TERESA GASSO, SALVADOR HERNÁNDEZ, AND ESPTIBEN ROJAS
}

\begin{abstract}
Let $X$ be a topological space and let $C(X)$ be the ring of all real-valued continuous functions defined on $X$. In this paper, we study the representation and approximation of continuous functions by sums of infinite series. Among other results, we give sufficient conditions in order to represent or approximate every continuous function by infinite series of functions, belonging to a previously fixed subfamily of $C(X)$, when $X$ is either a locally compact paracompact space or a Lindelöf space.
\end{abstract}

\section{INTRODUCTION}

Given a topological space, $X$, we denote by $C(X)$ the set of all real-valued continuous functions defined on $X$. The celebrated Stone-Weierstrass theorem yields very simple and easily verifiable conditions for the approximation of continuous functions defined on a compact space with respect to the uniform convergence topology. This result has been extended in different ways in order to obtain sufficient conditions for the approximation of bounded continuous functions spaces on general topological spaces. It is natural to ask whether it is possible to obtain analog results on the approximation of arbitrary, not necessarily bounded, continuous functions. Nevertheless, the approximation of unbounded continuous functions for the topology of uniform convergence is a difficult goal and it seems essential to include some appropriate tool that allows one to pass from local properties, which are given in terms of points and their neighborhoods, to global results, which concern the approximation of unbounded functions. A way of attacking the problem consists in considering the inversion operation of functions. Another possibility is to approximate through series of continuous functions (see $[10,8,9]$ ), which is the direction we deal with here. Indeed, the representation and approximation of continuous maps through infinite series of functions belonging to a previously fixed subset of $C(X)$ provides a method which is based on relatively simple operations and extends the well known theory about the approximation of continuous functions defined on subspaces of $\mathbb{R}^{n}$ by series of polynomials. Along this line, and using mainly topological methods, we present here some sufficient conditions in order to represent every continuous function as the sum

\footnotetext{
$1991 \quad$ Mathematics Subject Classification. Primary 54C30,54C40; Secondary 54C20,54D60,41A65,46E25.

Key words and phrases. Approximation by series of functions, Separation, S-separation, paracompact locally compact space, Lindelöf space, Lindelöf locally compact space, separable metrizable space.

The second named author acknowledges partial financial support by the Spanish Ministry of Science (including FEDER funds), grant MTM2004-07665-C02-01; and the Generalitat Valenciana, grant GV04B-019 .
} 
of an infinite series of functions, belonging to a previously determined subfamily $E$ of $C(X)$, when the topological space $X$ is either locally compact and paracompact or a Lindelöf space (recall that every separable metrizable space is Lindelöf). Our starting point has been a version of the classical Stone-Weierstrass theorem, given in [3], where it is made use of appropriately defined finite sums in order to obtain approximation results. This approach is also considered in $[10,2,5,6,7]$ where topological methods are also applied.

\section{Preliminaries}

$\mathbb{N}$ (resp. $\mathbb{R}$ ) denotes the set of all natural numbers (resp. real numbers). Throughout $X$ is a non void completely regular Hausdorff space. We denote by $C(X)$ (resp. $\left.C^{*}(X)\right)$ to the set of all real-valued continuous (resp. and bounded) functions defined on X. If $f \in C^{*}(X)$ we denote $\|f\|=\sup \{|f(x)|: x \in X\}$. For $A \subseteq X$ and $f \in C(X)$ we write $f_{\mid A}$ for the restriction of $f$ to $A$. If $E \subset C(X)$, then $E_{\mid A}=\left\{f_{\mid A}: f \in E\right\}$. Finally $\|f\|_{A}=\sup \{|f(x)|: x \in A\}$. Let $f \in C(X)$, we denote by supp $f$ the closure in $X$ of the set $\operatorname{coz}(f)=\{x \in X: f(x) \neq 0\}$. A zero set in $X$ is a set of the form $Z(f)=\{x \in X: f(x)=0\}$ with $f \in C(X)$. The symbol $Z(X)$ denotes the family of all zero sets in X. Let $f$ be an element of $C(X)$, then $f^{+}=f \vee 0$ and $f^{-}=(-f) \vee 0$. If $a$ is a real number, we denoted by $L_{a}(f)$ (resp. $\left.L^{a}(f)\right)$ the set $\{x \in X: f(x) \leq a\}$ (resp. $\{x \in X: f(x) \geq a\}$ ). We refer to $L_{a}(f)$ and $L^{a}(f)$ as the Lebesgue sets of $f$. From [10] and [2] we take the following two definitions.

Suppose that $E \subseteq C(X)$ and $A$ and $B$ are disjoint subsets of $X$, we say that $\mathrm{E}$ separates $A$ and $B$ (cf. [10] ) in case there is $f \in E$ such that $0 \leq f \leq 1, f_{A}=0$ and $f_{B}=1$. We say that $E S$-separates $A$ and $B$ (cf. [2]) when for each $\delta>0$ there is $f \in E$ such that $0 \leq f \leq 1, f(A) \subseteq[0, \delta]$ and $f(B) \subseteq[1-\delta, 1]$. We say that $E$ separates $f$ (resp. S-separarates $f$ ) if for every $a \leq b$ we have that $E$ separates (resp. $S$ - separates) the Lebesgue sets $L_{a}(f)$ and $\bar{L}^{b}(f)$. We need one more definition in order to distinguish these two separation conditions from the one used in the Stone Weierstrass theorem. We say that $E$ weakly separates $A$ and $B$ when there is $f \in E$ such that $f(A)=\{0\}$ and $f(B)=\{1\}$. Finally, here $E^{*}$ denotes the set $\{f \in E: f$ is bounded $\}$ and $\bar{E}^{u}$ denotes the uniform closure of $E$; i.e., the closure of $E$ in the topology of uniform convergence.

Given a series of continuous functions $\sum_{i \in I} f_{i}$ on $X$, we say that the series is locally convergent when, for every $x \in X$, there is a neighborhood $U$ of $x$ such that the series $\sum_{i \in I} f_{i}$ converges uniformly on $U$. Using an argument of compactness, it is readily seen that local convergence implies convergence in the compact open topology and that both definitions of convergence agree if $X$ is locally compact. For $E \subseteq C(X)$ we denote by $\sum(E)$ the set of all $f \in C(X)$ such that $f=\sum_{i \in I} f_{i}$ with $f_{i} \in E$ for every $i \in I$ and $\sum_{i \in I} f_{i}$ is a locally convergent series.

\section{BAsic RESUlts}

Lebesgue sets provide a natural and powerful technique, introduced by Lebesgue himself, in the approximation of functions, continuous or not. Next follows a basic simple result along this direction. 
Proposition 3.1. Let $X$ be a topological space and let $E$ be a vector subspace of $C(X)$. If $E S$-separates $f \in C(X)$, then $f$ and $|f|$ both belong to $\overline{\sum(E)}^{u}$.

Proof. Take $\delta>0$ arbitrary and suppose that $f \geq 0$. We define $F_{n}=L_{(n-1) \delta}(f)$ and $H_{n}=L^{n \delta}(f)$ for every $n \in \mathbb{N}$. By hypothesis, there is $\alpha_{n} \in E$ such that $0 \leq \alpha_{n} \leq 1, \alpha_{n}\left(F_{n}\right) \subset\left[0, \delta / 2^{n}\right]$ and $\alpha_{n}\left(H_{n}\right) \subset\left[1-\delta / 2^{n}, 1\right]$. The series $\sum_{n \in \mathbb{N}} \delta \alpha_{n}$ is locally convergent.

Indeed, take $x \in X$ with $n \delta \leq f(x)<(n+1) \delta$ and set $U=X \backslash L^{(n+1) \delta}(f)$, which is an open neighborhood of $x$. Then, for every $y \in U$, there is $n_{0} \in \mathbb{N}$ such that $y \in F_{n}$ for all $n \geq n_{0}$. So we have $\sum_{n \in \mathbb{N}} \delta \alpha_{n}(y)=\sum_{j=1}^{n_{0}-1} \delta \alpha_{j}(y)+\sum_{j=n_{0}}^{\infty} \delta \alpha_{j}(y)$. Since $\left\|\delta \alpha_{j}\right\|_{U} \leq \delta^{2} / 2^{j}$ for all $j \geq n_{0}$, we can apply the Weierstrass test in order to obtain that the series converges uniformly on $U$. Then

Now, in order to evaluate $\left|f(x)-\sum_{n \in \mathbb{N}} \delta \alpha_{n}(x)\right|$, suppose that $n \delta \leq f(x)<(n+1) \delta$.

$$
\sum_{j=1}^{n} \delta \alpha_{j}(x) \geq \delta \sum_{j=1}^{n}\left(1-\delta / 2^{j}\right) \geq n \delta-\delta^{2},
$$

and

So

$$
\sum_{j=n+2}^{\infty} \delta \alpha_{j}(x) \leq \delta^{2} / 2^{n+1}
$$

$$
\left|f(x)-\sum_{n \in \mathbb{N}} \delta \alpha_{n}(x)\right| \leq \delta+\delta^{2}
$$

Taking $\delta=\min (\epsilon / 2,1)$, we have

$$
\left\|f(x)-\sum_{n \in \mathbb{N}} \delta \alpha_{n}\right\|<\epsilon
$$

for any positive real number $\epsilon$. Hence, we are done when $f \geq 0$. If $f$ is not necessarily positive we have $f=f^{+}-f^{-}$(resp. $|f|=f^{+}+f^{-}$) and it is readily seen that $E$ $S$-separates $f^{+}$and $f^{-}$. Therefore, $f^{+}$and $f^{-}$belong to $\overline{\sum(E)}^{u}$, which completes the proof.

Remark 3.2. The following assertions are consequence of Proposition 3.1:

(1) We can use the decomposition $f=f^{+}-f^{-}$in order to approximate $f$ by functions $g \in \sum(E)$ such that $g=\sum_{n \in \mathbb{Z}} g_{n}, g_{n} \in E$ for all $n \in \mathbb{Z}$.

(2) Let $A$ be a subset of $C(X)$ and $E$ a vector subspace of $C(X)$ which $S$-separates Lebesgue sets of $A$. Then the sublattice generated by $A$ is contained in $\overline{\sum(E)}^{u}$.

(3) If we assume that $f$ is bounded and $E$ is a subalgebra of $C(X)$ in Proposition 3.1, then it follows easily that $f \in \bar{E}^{u}$ (cf. [2]).

(4) If $X$ is compact (or, even Lindelöf), then only countably indexed series are relevant in the definition of $\sum(E)$.

Proof. Items (1), (2), and (3) are readily seen. As for item (4), consider $f \in \sum(E)$, then $f=\sum_{i \in I} f_{i}$ with $f_{i} \in E$ for all $i \in I$. Since the series is locally convergent, for every $x \in X$ there is a neighborhood, $U_{x}$, of $x$ such that for a countable subset $J_{x} \subset I$, we have $f_{i \mid U_{x}}=0$ if $i \notin J_{x}$. By an argument of compactness, it is easy to see 
that there is a countable subset of $J \subset I$, such that $f_{i}=0$ if $i \notin J$. That is to say, only countably indexed series are relevant in the definition of $\sum(E)$.

\section{LOCALly COMPACT PARACOMPACT SPACES}

In this section we present some results which relate local with global approximation properties for locally compact paracompact spaces. Firstly, we present some preliminaries results that will be needed in the sequel.

Lemma 4.1. Let $X$ a be a topological space and let $E$ be a vector sublattice of $C(X)$ which contains the constants. If $A$ and $B$ are two subsets of $X$ that are $S$-separated by $E$, then they are separated by $E$.

Proof. Let $g \in E$ with $0 \leq g \leq 1, g_{\mid A} \leq \delta$ and $g_{\mid B} \geq 1-\delta$ where $0<\delta<\frac{1}{2}$. Define $\left.f=\left[\frac{1}{1-2 \delta} \cdot((g-\delta) \vee 0)\right)\right] \wedge 1$. Then $0 \leq f \leq 1, f_{\mid A}=0$ and $f_{\mid B}=1$.

Next, follows a variant of Proposition 1 which relaxes the conditions of S-separation when we have partitions of the unity.

Lemma 4.2. Let $X$ be a topological space and $E$ a subalgebra of $C(X)$. Let $f \in C(X)$ be such that there is a locally finite partition of the unity $\left\{\alpha_{i}\right\}_{i \in I}$ with $\alpha_{i} \in E, i \in I$, and $E_{\mid c o z\left(\alpha_{i}\right)}$ S-separates $f_{\mid c o z\left(\alpha_{i}\right)}$ for all $i \in I$. Then $f \in \overline{\sum(E)}^{u}$

Proof. Firstly, we set $A_{i}=\operatorname{coz}\left(\alpha_{i}\right)$ in order to simplify the notation. Given an arbitrary but fixed $\epsilon>0$, since $E_{\mid A_{i}}$ S-separates $f_{\mid A_{i}}$, for every $\delta>0$ there is $g_{i} \in E$ such that $0 \leq g_{i \mid A_{i}} \leq 1, \quad g_{i \mid\left(L_{a}(f) \cap A_{i}\right)}<\delta$ and $g_{i \mid\left(L^{b}(f) \cap A_{i}\right)}>1-\delta$. Now, the map

$$
g=\sum_{i \in I} \alpha_{i} g_{i}
$$

satisfies the following three conditions:

(1) $0 \leq g \leq 1$

(2) if $x \in L^{b}(f)$ then

$$
g(x)=\sum \alpha_{i}(x) g_{i}(x)>\sum \alpha_{i}(x)(1-\delta)=1-\delta
$$

(3) If $x \in L_{a}(f)$ then

$$
g(x)=\sum \alpha_{i}(x) g_{i}(x)<\sum \alpha_{i}(x) \delta<\delta .
$$

This means that $\sum(E)$ is a vector subspace of $C(X)$ that S-separates $L_{a}(f)$ and $L^{b}(f)$ for all $a<b$. Therefore, by Proposition 1, for every $\epsilon>0$ there is a locally convergent series $\sum_{n \in \mathbb{Z}} h_{n}$ such that

$$
h_{n}=\sum_{i \in I} \alpha_{i} g_{i_{n}} \quad \text { and } \quad\left\|f-\sum_{n \in \mathbb{Z}} h_{n}\right\|<\epsilon .
$$

In order to finish the proof, it will suffice to prove that

$$
\sum h_{n}=\sum_{n \in \mathbb{Z}, i \in I} \alpha_{i} g_{i_{n}}
$$

is locally convergent. 
Now, let $x$ be an arbitrary element of $X$. Since $\sum_{n \in \mathbb{Z}} h_{n}$ is locally convergent, there is a neighborhood $U$ of $x$ such that $\sum_{n \in \mathbb{Z}} h_{n \mid U}$ is uniformly convergent. On the other hand, the family $\left\{A_{i}: i \in I\right\}$ is locally finite, therefore, there is another neighborhood $V$ of $x$ and a finite subset $J$ of $I$ such that $V \cap A_{i}=\emptyset$ for all $i \in I \backslash J$. So we have

$$
\sum_{n \in \mathbb{Z}} h_{n \mid(U \cap V)}=\sum_{n \in \mathbb{Z}, i \in J}\left(\alpha_{i} g_{i}\right)_{\mid U \cap V}=\sum_{n \in \mathbb{Z}, i \in I}\left(\alpha_{i} g_{i}\right)_{\mid U \cap V}
$$

Since $J$ is finite and $\sum_{n \in \mathbb{Z}} h_{n \mid U}$ is uniformly convergent, it follows that $\sum_{n \in \mathbb{Z}, i \in I} \alpha_{i} g_{i_{n}}$ converges uniformly on $U \cap V$, which completes the proof.

Next result is a variation of the Stone Weierstrass theorem for locally compact Lindelöf spaces.

Lemma 4.3. Let $X$ be a locally compact Lindelöf space and let $E$ be a subalgebra of $C(X)$ which contains the constants and weakly separates points of $X$. Then $\sum(E)=$ $C(X)$.

Proof. Let $\left\{A_{n}: n \in \mathbb{N}\right\}$ be a sequence of compact sets in $X$ such that $A_{n} \subseteq$ int $A_{n+1}$ for every $n \in \mathbb{N}$ and $\bigcup\left\{A_{n}: n \in \mathbb{N}\right\}=X$. Given $\varepsilon>0$, since $E$ weakly separates points in $\mathrm{X}$, the Stone-Weierstrass theorem yields the existence of a map $f_{1} \in E$ such that $\left\|f-f_{1}\right\|<\frac{\varepsilon}{2}$. Let $g_{1}=f-f_{1}$, repeating the same argument, there is a $f_{2} \in E$ such that $\left\|g_{1}-f_{2}\right\|_{A_{2}}<\frac{\varepsilon}{2^{2}}$ or equivalently $\left\|f-\left(f_{1}+f_{2}\right)\right\|_{A_{2}}<\frac{\varepsilon}{2^{2}}$. In general, let $g_{n}=f-\left(f_{1}+f_{2}+\cdots+f_{n}\right)$ with $\left\|g_{n}\right\|_{A_{n}}<\frac{\varepsilon}{2^{n}}$, then there is $f_{n+1} \in E$ such that $\left\|g_{n}-f_{n+1}\right\|_{A_{n+1}}<\frac{\varepsilon}{2^{n+1}}$ or, equivalently, $\left\|f-\left(f_{1}+\cdots+f_{n+1}\right)\right\|_{A_{n+1}}<\frac{\varepsilon}{2^{n+1}}$. Reasoning by induction, we obtain a sequence $\left\{f_{n}: n \in \mathbb{N}\right\}$ in $E$ such that the series $\sum_{n \in \mathbb{N}} f_{n}$ is locally convergent and $f(x)=\sum_{n \in \mathbb{N}} f_{n}$ for all $x \in X$. This completes the proof.

Corollary 4.4. Every real-valued continuous function defined on $\mathbb{R}^{n}$ can be expressed as the sum of locally convergent series of polynomials.

Corollary 4.5. Let $X$ be a Tychonoff space and let $E$ be a subalgebra os $C(X)$ such that $E$ contains the constants and $E^{*}$ weakly separates zero-sets in $X$. Then $\sum(E)=C(X)$.

Proof. Let $f \in C(X)$ be arbitrarily chosen. By the universal property of the StoneCech compactification $\beta X$, the map $f$ can be extended to a continuous function $\widetilde{f}: \beta X \longrightarrow \mathbb{R} \cup \infty$. Let $Y=\beta X \backslash f^{-1}(\infty)$ and $\widehat{f}=\widetilde{f}_{\mid Y}$. We have that $Y$ is a locally compact Lindelöf space and $\widehat{f} \in C(Y)$. Define $\widehat{E}=\left\{\widehat{g}: g \in E\right.$ and $\widetilde{g}_{\mid Y}$ is real valued $\}$. Since $E^{*}$ weakly separates $Z(X)$, we deduce that $\widehat{E}$ weakly separates points in $Y$. By Lemma 4.3 , there is a locally convergent series, $\sum_{n \in \mathbb{N}} \widehat{g}_{n}$ with $g_{n} \in E$ for all $n \in \mathbb{N}$, such that $\widehat{f}=\sum_{n \in \mathbb{N}} \widehat{g}_{n}$. Therefore $f=\sum_{n \in \mathbb{N}} g_{n}$ and we are done.

Corollary 4.6. Let $X$ and $Y$ be two topological spaces such that $X \times Y$ is locally compact and Lindelöf. Then, for every $f \in C(X \times Y)$, there are sequences $\left\{f_{i}: i \in\right.$ $\mathbb{N}\} \subseteq C(X),\left\{g_{i}: i \in \mathbb{N}\right\} \subseteq C(Y)$, and $\left\{n_{k}: k \in \mathbb{N}\right\} \subseteq \mathbb{N}$ such that

$$
f(x, y)=\sum_{k \in \mathbb{N}}\left[\sum_{n_{k} \leq i<n_{k+1}} f_{i}(x) g_{i}(y)\right]
$$

for all $(x, y) \in X \times Y$, and the series is locally convergent. 
Proof. Let $C(X) \otimes C(Y)=\left\{\sum_{i=1}^{n} f_{i} g_{i}: f_{i} \in C(X), g_{i} \in C(Y), n \in \mathbb{N}\right\}$. Then $C(X) \otimes C(Y)$ separates points in $X \times Y$ and it suffices to apply Lemma 4.3.

We now prove the principal approximation result for locally compact paracompact spaces.

Theorem 4.7. Let $X$ be a locally compact paracompact space and let $E$ be a subalgebra of $C(X)$ which is a lattice, contains the constants, and weakly separates points of $X$. Then $\sum(E)=C(X)$.

Proof. By [4, Th. 5.1.27], it follows that there exists a family $\left\{X_{i}: i \in I\right\}$ of pairwise disjoint clopen Lindelöf subspaces of $X$ such that $X=\cup_{i \in I} X_{i}$. For each $i \in I$, let $\left\{A_{n, i}: n \in \mathbb{N}\right\}$ be a sequence of compact subsets of $X$ such that $\cup_{n \in \mathbb{N}} A_{n, i}=X_{i}$ and $A_{n, i} \subset \operatorname{int}_{X} A_{(n+1), i}$ for any $n \in \mathbb{N}, i \in I$. We shall inductively define a partition of the unity $\left\{\alpha_{n, i}: n \in \mathbb{N}, i \in I\right\} \subset E$ such that

$$
\left\|1-\sum_{k=1}^{n} \alpha_{k, i}\right\|_{A_{n, i}} \leq \frac{1}{2^{n}} \quad \text { and } \quad \operatorname{coz}\left(\alpha_{n, i}\right) \subset X_{i} .
$$

Indeed, by the Stone-Weierstrass theorem, there are $g_{1, i} \in E$ such that $\left\|1-g_{1, i}\right\|_{A_{1, i}} \leq$ $\frac{1}{2}$. Applying the argument of Lemma 4.1, there exists $r_{1, i} \in E$ such that $r_{1, i}\left(A_{1, i}\right)=$ $\{1\}$ and $\operatorname{coz}\left(r_{1, i}\right) \subset X_{i}$. Put $\alpha_{1, i}=r_{1, i} g_{1, i}$. Suppose that, for any $i \in I$, we have already defined functions $\left\{\alpha_{1, i}, \ldots, \alpha_{n, i}\right\}$ having property (1). Using the StoneWeierstrass theorem and Lemma 4.1 again, we obtain that there exist functions $g_{n+1, i}$ and $r_{n+1, i}$ in $E$ such that

$$
\left\|\left(1-\sum_{k=1}^{n} \alpha_{k, i}\right)-g_{n+1, i}\right\|_{A_{n+1, i}} \leq \frac{1}{2^{n+1}}, r_{n+1, i}\left(A_{n+1, i}\right)=\{1\} \text { and } \operatorname{coz}\left(r_{n+1, i}\right) \subset X_{i} .
$$

Define $\alpha_{n+1, i}=r_{n+1, i} \cdot g_{n+1, i}$. The family $\left\{\alpha_{n, i}\right\}$ is a partition of the unity such that $\operatorname{coz}\left(\alpha_{n, i}\right)$ is locally compact and Lindelöf for all pair $n, i$. Applying Lemma 4.3, we have that

$$
\sum(E)_{\mid \operatorname{coz}\left(\alpha_{n, i}\right)}=C(X)_{\mid \operatorname{coz}\left(\alpha_{n, i}\right)}
$$

Reasoning as in the proof of Lemma 4.2, it follows that $f \in \sum(E)$, which completes the proof.

As a consequence we obtain the representation of every continuous map in a product space as the sum of a series formed by finite sums of products of functions which depend of only one variable. Here, given an arbitrary set $S$, we denote by $[S]^{<\omega}$ the collection of all finite subsets of $S$.

Corollary 4.8. Let $X$ and $Y$ be two topological spaces such that $X \times Y$ is locally compact and paracompact. Then, for every $f \in C(X \times Y)$, there are $\left\{f_{i}: i \in I\right\} \subseteq$ $C(X),\left\{g_{j}: j \in J\right\} \subseteq C(Y)$, and a directed subset $\mathcal{D}$ of $[I \times J]^{<\omega}$ such that

$$
f(x, y)=\sum_{D \in \mathcal{D}}\left[\sum_{(i, j) \in D} f_{i}(x) \cdot g_{j}(y)\right]
$$

for all $(x, y) \in X \times Y$, and the series is locally convergent. 
Proof. Let $C(X) \otimes C(Y)=\left\{\sum_{i=1}^{n} f_{i} g_{i}: f_{i} \in C(X), g_{i} \in C(Y), n \in \mathbb{N}\right\}$. Obviously $C(X) \otimes C(Y)$ is a subalgebra of $C(X \times Y)$ which separates points in $X \times Y$.

Let $\left\{X_{i}: i \in I\right\}$ and $\left\{Y_{j}: i \in J\right\}$ be families of pairwise disjoint clopen Lindelöf subspaces of $X$ and $Y$ respectively such that $X=\cup_{i \in I} X_{i}$ and $Y=\cup_{j \in J} Y_{j}$. For each $i \in I$ and $j \in J)$ let $\left\{A_{n, i}: n \in \mathbb{N}\right\}$ and $\left\{B_{n, j}: n \in \mathbb{N}\right\}$ be sequences of compact subsets of $X$ and $Y$ respectively such that $\cup_{n \in \mathbb{N}} A_{n, i}=X_{i}, A_{n, i} \subset i n t_{X} A_{(n+1), i}$ for any $n \in \mathbb{N}, i \in I$ and $\cup_{n \in \mathbb{N}} B_{n, j}=Y_{j}, B_{n, j} \subset$ int $_{Y} B_{(n+1), j}$ for any $n \in \mathbb{N}, j \in J$. We shall inductively define a partition of the unity $\left\{\alpha_{n, i, j}: n \in \mathbb{N}, i \in I, j \in J\right\} \subset C(X) \otimes C(Y)$ such that

$$
\left\|1-\sum_{k=1}^{n} \alpha_{k, i, j}\right\|_{A_{n, i} \times B_{n, j}} \leq \frac{1}{2^{n}} \quad \text { and } \quad \operatorname{coz}\left(\alpha_{n, i, j}\right) \subset X_{i} \times Y_{j} .
$$

Indeed, by the Stone-Weierstrass theorem, there is $g_{1, i, j} \in C(X) \otimes C(Y)$ such that $\left\|1-g_{1, i, j}\right\|_{A_{1, i} \times B_{1, j}} \leq \frac{1}{2}$. Clearly, there exist $r_{1, i} \in C(X)$ and $s_{1, j} \in C(Y)$ such that $r_{1, i}\left(A_{1, i}\right)=\{1\}, \operatorname{coz}\left(r_{1, i}\right) \subset X_{i}$ and $s_{1, j}\left(B_{1, j}\right)=\{1\}, \operatorname{coz}\left(s_{1, j}\right) \subset Y_{j}$. Set $\alpha_{1, i, j}=$ $r_{1, i} s_{1, j} g_{1, i, j} \in C(X) \otimes C(Y)$. Suppose that, for any $(i, j) \in I \times J$, we have already defined functions $\left\{\alpha_{1, i, j}, \ldots, \alpha_{n, i, j}\right\}$ having property (2). Using the Stone-Weierstrass theorem again, we obtain that there exist functions

$$
g_{n+1, i, j} \in C(X) \otimes C(Y), r_{n+1, i} \in C(X), s_{n+1, j} \in C(Y)
$$

such that

$$
\begin{gathered}
\left\|\left(1-\sum_{k=1}^{n} \alpha_{k, i, j}\right)-g_{n+1, i, j}\right\|_{A_{n+1, i} \times B_{n+1, j}} \leq \frac{1}{2^{n+1}}, \\
r_{n+1, i}\left(A_{n+1, i}\right)=\{1\}, s_{n+1, j}\left(B_{n+1, j}\right)=\{1\}
\end{gathered}
$$

and

$$
\operatorname{coz}\left(r_{n+1, i}\right) \subset X_{i}, \operatorname{coz}\left(s_{n+1, j}\right) \subset Y_{j} .
$$

Define $\alpha_{n+1, i, j}=r_{n+1, i} s_{n+1, j} g_{n+1, i, j} \in C(X) \otimes C(Y)$. The family $\left\{\alpha_{n, i, j}\right\}$ is a partition of the unity such that $\operatorname{coz}\left(\alpha_{n, i, j}\right)$ is locally compact and Lindelöf for all triple $(n, i, j)$. Applying Lemma 4.3, we have that

$$
\sum(C(X) \otimes C(Y))_{\mid \operatorname{coz}\left(\alpha_{n, i, j}\right)}=C(X \times Y)_{\mid \operatorname{coz}\left(\alpha_{n, i, j}\right)}
$$

Reasoning as in the proof of Lemma 4.2, it follows that $f \in \sum(C(X) \otimes C(Y))$, which completes the proof.

Obviously, this result can be extended to any finite product. On the other hand, we have been informed by Professor Bierstedt that Corollaries 4.6 and 4.8 can also be obtained using the fact that, when $X$ and $Y$ are $k_{\mathbb{R}}$ spaces, the $\epsilon$-completion of the space $C(X) \otimes C(Y)$ coincides with $C(X \times Y)$ (see [1, Cor. 35]).

\section{Approximation in LindelÖF SPACES}

We are concerned in this Section with spaces which are not necessarily locally compact. In the absence of local compactness, the techniques, which we have used so far, do not work even for spaces with very nice properties. In this Section, using more involved arguments, we present some results on the approximation of continuous functions for Lindelöf spaces. The proofs could be modified in order to get some more 
general achievements but we have opted for a weaker version in order to simplify the technicalities.

Let $E$ be a vector subspace of $C(X)$, we say that $E$ locally $S$-separates zero sets when $E$ satisfies two properties:

(1) for every pair $Z_{1}, Z_{2} \in Z(X)$ with $Z_{1} \cap Z_{2}=\emptyset$ and every $x \in X$ there exists a neighborhood $U$ of $x$ such that $E S$-separates $Z_{1} \cap U$ and $Z_{2} \cap U$; and

(2) for every pair of subsets $C$ and $V$ such that $C \subset V \subset U$, with $C$ closed and $V$ open in $X$, there is $a \in E$ such that $0 \leq a \leq 1, a(C)>1-\delta$, and $a(X \backslash V)<\delta$.

Theorem 5.1. Let $X$ be a Lindelöf space and let $E$ be a subalgebra of $C(X)$, which contains the constants and locally separates zero sets. Then $C(X)=\overline{\sum(E)}^{u}$.

Proof. Let $f \in C(X)$ and $\epsilon>0$. If $L_{a}(f)$ and $L^{b}(f)$ are two disjoint Lebesgue sets of $f$, for every $x \in X$ there is $U_{x} \in \mathcal{N}(x)$ such that $E$ separates $L_{a}(f) \cap U_{x}$ and $L^{b}(f) \cap U_{x}$. Consider the family $\left\{U_{x}: x \in X\right\}$ and let $\left\{A_{i}: i \in I\right\}$ be a family of open subsets which is a locally finite open refinement of $\left\{U_{x}: x \in X\right\}$. Since $X$ is a Lindelöf space, we can assume that the family $\left\{A_{i}: i \in I\right\}$ is star-finite. That is to say, every $A_{j}$ intersects finitely many elements, say $n_{j}$, of the family $\left\{A_{i}: i \in I\right\}$ (see [11, V.4 B)]). Now, since $E_{\mid A_{i}} S$-separates $L_{a}(f) \cap A_{i}$ and $L^{b}(f) \cap A_{i}$, there is $h_{i} \in E$ such that $h_{i}\left(L_{a}(f) \cap A_{i}\right) \subset[0, \epsilon), h_{i}\left(L^{b}(f) \cap A_{i}\right) \subset(1-\epsilon, 1]$ and $0 \leq h_{i} \leq 1$. Let $\left\{\alpha_{i}: i \in I\right\}$ be a partition of the unity subordinated to the cover $\left\{A_{i}: i \in I\right\}$ and let $j \in I$ be arbitrarily chosen. As $E S$-separates $Z(X) \cap A_{i}$ and every $\alpha_{j}$ is bounded, applying Proposition 3.1, it follows that $\alpha_{j}$ can be approximated by functions of $E_{\mid A_{j}}$. Therefore, there is $\beta_{j} \in E$ such that $0 \leq \beta_{j} \leq 1$ and $\left\|\alpha_{j}-\beta_{j}\right\|_{A_{j}}<\epsilon / n_{j}$. In like manner, using item (2) in the definition of local $S$-separation, there is $r_{j} \in E$ such that $0 \leq r_{j} \leq 1, r_{j}\left(\operatorname{supp} \alpha_{j}\right) \subset\left(1-\epsilon / n_{j}, 1\right]$ and $r_{j}\left(X \backslash A_{j}\right) \subset\left[0, \epsilon / n_{j}\right)$. We define the function

$$
\phi=\frac{1}{1+\epsilon} \sum_{i \in I} \beta_{i} r_{i} h_{i} .
$$

It is clear that $\phi \geq 0$. On the other hand, suppose that $x$ is an arbitrary element of $A_{j}$, then $A_{j}$ intersects a finite subfamily, say $\left\{A_{i_{1}}, \ldots, A_{i_{n_{j}}}\right\}$. Then

$$
\phi(x) \leq \frac{1}{1+\epsilon} \sum_{1 \leq k \leq n_{j}} \beta_{i_{k}}(x) \leq \frac{1}{1+\epsilon} \sum_{1 \leq k \leq n_{j}}\left(\alpha_{i_{k}}(x)+\frac{\epsilon}{n_{j}}\right)=1 .
$$

Therefore, $0 \leq \phi \leq 1$. Finally, suppose that $x$ is a point in $A_{j} \cap L^{b}(f)$, then

$$
\begin{gathered}
\phi(x)=\frac{1}{1+\epsilon} \sum_{1 \leq k \leq n_{j}}\left(\beta_{i_{k}} r_{i_{k}} h_{i_{k}}\right)(x) \geq \\
\frac{1}{1+\epsilon} \sum_{1 \leq k \leq n_{j}}\left\{\left(\alpha_{i_{k}} r_{i_{k}} h_{i_{k}}\right)(x)-\frac{\epsilon}{n_{j}}\left(r_{i_{k}} h_{i_{k}}\right)(x)\right\} \geq \\
\frac{1}{1+\epsilon} \sum_{1 \leq k \leq n_{j}}\left\{\alpha_{i_{k}}(x)(1-\epsilon)^{2}-\frac{\epsilon}{n_{j}}\right\} \geq \\
\frac{(1-\epsilon)^{2}-\epsilon}{1+\epsilon}=l_{1}(\epsilon) .
\end{gathered}
$$


In the same way, if $x$ belongs to $A_{j} \cap L_{a}(f)$

$$
\begin{gathered}
\phi(x) \leq \frac{1}{1+\epsilon} \sum_{1 \leq k \leq n_{j}}\left\{\left(\alpha_{i_{k}} r_{i_{k}} h_{i_{k}}\right)(x)+\frac{\epsilon}{n_{j}}\left(r_{i_{k}} h_{i_{k}}\right)(x)\right\} \leq \\
\frac{1}{1+\epsilon} \sum_{1 \leq k \leq n_{j}}\left\{\left(\alpha_{i_{k}} r_{i_{k}}\right)(x) \epsilon+\frac{\epsilon}{n_{j}} r_{i_{k}}(x) \epsilon\right\} \leq \\
\frac{\epsilon+\epsilon^{2}}{1+\epsilon}=l_{2}(\epsilon) .
\end{gathered}
$$

Since $\lim _{\epsilon \rightarrow 0} l_{1}(\epsilon)=1$ and $\lim _{\epsilon \rightarrow 0} l_{2}(\epsilon)=0$, we have proved that, for every $\rho>0$ there is a map

$$
\phi_{(a, b, \rho)}=\sum_{i \in I} g_{i}
$$

such that $g_{i} \in E$ for all $i \in I$ such that $0 \leq \phi_{(a, b, \rho)} \leq 1, \phi_{(a, b, \rho)}\left(L_{a}(f)\right) \subset[0, \rho)$, and $\phi_{(a, b, \rho)}\left(L^{b}(f)\right) \subset(1-\rho, 1]$. Furthermore, the map $\phi_{(a, b, \rho)}$ is defined by a finite sum on every $A_{j}, j \in I$. Now, for every $\epsilon$ with $0<\epsilon<1$ and $n \in \mathbb{N}$, set

$$
a_{n}=\frac{(n-1) \epsilon}{2}, \quad b_{n}=\frac{n \epsilon}{2} \quad \text { and } \quad \rho_{n}=\frac{\epsilon}{2^{n+1}}
$$

We obtain a sequence of maps

$$
\phi_{\left(a_{n}, b_{n}, \rho_{n}\right)}=\sum_{i \in I} g_{n i}, \quad n \in \mathbb{N}
$$

Now, it suffices to observe that the function

$$
\phi=\sum_{i \in I, n \in \mathbb{N}} \frac{\epsilon}{2} g_{n i}
$$

belongs to $E$ and $\|f-\phi\|<\epsilon$. This completes the proof.

\section{Acknowledgment}

We wish to thank Professor Bierstedt for his helpful comments on a previous version of this paper.

\section{REFERENCES}

[1] K. Bierstedt, Tensor Products of Weighted Spaces, Bonner Math. Schriften 81 1975, 26-58.

[2] J.L. Blasco and A. Moltó, On the Uniform Closure of a Linear Space of Bounded Real-Valued Functions, Annali di Matematica pura ed applicata 134 (4) 1983, 233-239.

[3] B. Brosowski and F. Deutsch, An Elementary Proof of the Stone-Weierstrass Theorem, PAMS, 81 (1) 1981, 89-92.

[4] R. Engelking, General Topology, Polish Scientific, Warszawa, 1977.

[5] M.I. Garrido and F. Montalvo, Generation of the uniformly continuous functions, Topology and its Applications, 137 2004, 167174. 
[6] M.I. Garrido and F. Montalvo, Generation of uniformly closed algebras of functions, Positivity, $92005,8195$.

[7] F. Montalvo, A. Pulgarín and B. Requejo, Indag. Mathem. N.S.17 (2) 2006, 285-295.

[8] A. Hager, On inverse-closed subalgebras of $C(X)$, Proc. London Math. Soc, III-19 1969, 233257.

[9] A. Hager, An approximation technique for real-valued functions, General Topology and its Applications, 1 1971, 127-133.

[10] S. Mrowka, On some approximation theorems, Nieuw Arch. Wisk., 16 1968, 94-111.

[11] J. Nagata, Modern General Topology, North-Holland, Amsterdam, and Wolters-noordhoff, Groningen, 1968.

Departmento de Matemáticas,

Universidad PolitéCnica de VAlencia,

FACUltad de InformáticA, VALEnCia 46022,

U.S.A.

E-mail address: mgassó@mat.upv.es

Departamento de Matemáticas,

Área Científico-TÉCNICA,

UNIVERSIDAD JAUME I,

8029 - AP CASTELLÓN,

SPAIN.

E-mail address: hernande@mat.uji.es

Departamento de Ciencias Exactas,

UNIVERSIDAD DE LOS LAGOS,

OSORNO,

CHILE.

E-mail address: esptiben@mat.puc.cl 